\title{
Vorapaxar in the Secondary Prevention of Atherothrombotic Events
}

\author{
David A. Morrow, M.D., M.P.H., Eugene Braunwald, M.D., Marc P. Bonaca, M.D., \\ Sebastian F. Ameriso, M.D., Anthony J. Dalby, M.B., Ch.B., Mary Polly Fish, B.A., \\ Keith A.A. Fox, M.B., Ch.B., Leslie J. Lipka, M.D., Ph.D., Xuan Liu, Ph.D., \\ José Carlos Nicolau, M.D., Ph.D., A.J. Oude Ophuis, M.D., Ph.D., \\ Ernesto Paolasso, M.D., Benjamin M. Scirica, M.D., M.P.H., \\ Jindrich Spinar, M.D., Ph.D., Pierre Theroux, C.M., M.D., \\ Stephen D. Wiviott, M.D., John Strony, M.D., and Sabina A. Murphy, M.P.H., \\ for the TRA 2P-TIMI 50 Steering Committee and Investigators*
}

ABSTRACT

\begin{abstract}
The authors' affiliations are listed in the Appendix. Address reprint requests to Dr. Morrow at the TIMI Study Group, Cardiovascular Division, Brigham and Women's Hospital, 75 Francis St., Boston, MA 02115, or atdmorrow@partners.org.
\end{abstract}

*Members of the Thrombin Receptor Antagonist in Secondary Prevention of Atherothrombotic Ischemic Events (TRA 2P)-Thrombolysis in Myocardial Infarction (TIMI) 50 steering committee, as well as other committee members and investigators, are listed in the Supplementary Appendix, available at NEJM.org.

This article (10.1056/NEJMoal200933) was published on March 24, 2012, at NEJM.org.

N EngIJ Med 2012;366:1404-13.

Copyright ( 2012 Massachusetts Medical Society.

\section{BACKGROUND}

Thrombin potently activates platelets through the protease-activated receptor PAR-1. Vorapaxar is a novel antiplatelet agent that selectively inhibits the cellular actions of thrombin through antagonism of PAR-1.

\section{METHODS}

We randomly assigned 26,449 patients who had a history of myocardial infarction, ischemic stroke, or peripheral arterial disease to receive vorapaxar (2.5 mg daily) or matching placebo and followed them for a median of 30 months. The primary efficacy end point was the composite of death from cardiovascular causes, myocardial infarction, or stroke. After 2 years, the data and safety monitoring board recommended discontinuation of the study treatment in patients with a history of stroke owing to the risk of intracranial hemorrhage.

\section{RESULTS}

At 3 years, the primary end point had occurred in 1028 patients $(9.3 \%)$ in the vorapaxar group and in 1176 patients $(10.5 \%)$ in the placebo group (hazard ratio for the vorapaxar group, 0.87 ; $95 \%$ confidence interval $[\mathrm{CI}], 0.80$ to $0.94 ; \mathrm{P}<0.001$ ). Cardiovascular death, myocardial infarction, stroke, or recurrent ischemia leading to revascularization occurred in 1259 patients (11.2\%) in the vorapaxar group and 1417 patients $(12.4 \%$ ) in the placebo group (hazard ratio, 0.88 ; $95 \% \mathrm{CI}, 0.82$ to $0.95 ; \mathrm{P}=0.001$ ). Moderate or severe bleeding occurred in $4.2 \%$ of patients who received vorapaxar and $2.5 \%$ of those who received placebo (hazard ratio, 1.66; 95\% CI, 1.43 to 1.93; $\mathrm{P}<0.001)$. There was an increase in the rate of intracranial hemorrhage in the vorapaxar group $(1.0 \%$, vs. $0.5 \%$ in the placebo group; $\mathrm{P}<0.001)$.

\section{CONCLUSIONS}

Inhibition of PAR-1 with vorapaxar reduced the risk of cardiovascular death or ischemic events in patients with stable atherosclerosis who were receiving standard therapy. However, it increased the risk of moderate or severe bleeding, including intracranial hemorrhage. (Funded by Merck; TRA 2P-TIMI 50 ClinicalTrials.gov number, NCT00526474.) 
P LATELETS PLAY A CENTRAL ROLE IN ATHerothrombosis and are an important target for pharmacotherapy. In patients with acute coronary syndromes, the use of potent platelet inhibitors has been shown to reduce the rate of thrombotic events at the cost of increased bleeding. ${ }^{1-3}$ In contrast, among patients with stable atherosclerosis, a reduced rate of thrombotic events with antiplatelet therapy in addition to aspirin therapy has not been established. ${ }^{4}$

Thrombin is a serine protease that is critical in thrombosis. In addition to generating fibrin, thrombin is a potent agonist of platelets through interaction with protease-activated receptors (PARs). ${ }^{5}$ Vorapaxar (SCH 530348, Merck) is a competitive and selective antagonist of PAR-1, the major thrombin receptor on human platelets. Vorapaxar potently inhibits thrombin-induced platelet aggregation. $^{6,7}$ This study, called the Thrombin Receptor Antagonist in Secondary Prevention of Atherothrombotic Ischemic Events (TRA 2P)Thrombolysis in Myocardial Infarction (TIMI) 50 trial, was designed to evaluate the efficacy and safety of vorapaxar in reducing atherothrombotic events in patients with established atherosclerosis who were receiving standard therapy. In addition to investigating vorapaxar specifically, the trial more broadly tested the hypothesis that the intensification of antiplatelet therapy by adding an agent with a different pharmacologic target is beneficial for secondary prevention in patients with stable disease and a history of myocardial infarction, ischemic stroke, or peripheral arterial disease.

\section{METHODS}

\section{STUDY DESIGN AND OVERSIGHT}

The TRA 2P-TIMI 50 trial was a multinational, double-blind, placebo-controlled trial ${ }^{8}$ that was conducted at 1032 sites in 32 countries (see the Supplementary Appendix, available with the full text of this article at NEJM.org). The trial was sponsored by Merck and was designed by the TIMI Study Group in conjunction with the steering committee and trial sponsor. The protocol was approved by the relevant ethics committees at all participating centers. The raw database was provided to the TIMI Study Group, which carried out the data analyses independently of the sponsor, prepared this report, and made the decision to submit the manuscript for publication. The members of the TIMI Study Group assume responsibility for the accuracy and completeness of the data and all analyses and for the fidelity of this report to the study protocol, which is available at NEJM.org.

\section{STUDY POPULATION}

Eligible patients had a history of atherosclerosis, which was defined as a spontaneous myocardial infarction or ischemic stroke within the previous 2 weeks to 12 months or peripheral arterial disease associated with a history of intermittent claudication in conjunction with either an anklebrachial index of less than 0.85 or previous revascularization for limb ischemia. By design, enrollment of patients with a qualifying diagnosis of either stroke or peripheral arterial disease was to end when the number enrolled reached approximately $15 \%$ of the total anticipated sample size.

Patients were ineligible if they were planning to undergo a revascularization procedure, had a history of bleeding diathesis, had recent active abnormal bleeding, were receiving ongoing treatment with warfarin, or had active hepatobiliary disease. The full eligibility criteria have been reported previously. ${ }^{8}$ Written informed consent was obtained from all patients.

\section{RANDOMIZATION AND STUDY TREATMENT}

Eligible patients were randomly assigned in a 1:1 ratio to receive either vorapaxar ( $2.5 \mathrm{mg}$ daily) or matched placebo by a central computerized system with hierarchical stratification according to the qualifying diagnosis (myocardial infarction, stroke, or peripheral arterial disease) and the responsible physician's intent to administer a thienopyridine (see the Methods section in the Supplementary Appendix). Vorapaxar and placebo were administered orally in a blinded fashion once daily until the end of follow-up. Study therapy was to be interrupted if the patient required treatment with either a potent inhibitor of the cytochrome P-450 3A4 (CYP3A4) enzyme system or warfarin in conjunction with a thienopyridine. All concomitant medical therapy, including the use of other antiplatelet agents, was managed by the clinicians at the study sites who were responsible for the care of the patients, according to local standards of care.

In January 2011, after completion of enrollment and a median of 24 months of follow-up, the data and safety monitoring board reported an excess of intracranial hemorrhage in patients with a history of stroke in the vorapaxar group and recommended discontinuation of the drug in all patients with 
previous stroke, including those with a new stroke during the trial. The board also recommended continuation of the trial in patients without a history of stroke. The protocol was amended accordingly (see the Methods section in the Supplementary Appendix).

\section{END POINTS}

The protocol-defined primary efficacy end point was a composite of cardiovascular death, myocardial infarction, stroke, or recurrent ischemia leading to urgent coronary revascularization. The major secondary end point was a composite of cardiovascular death, myocardial infarction, or stroke. Before the database was locked and during blinded treatment, the investigators reviewed data from the newly completed Thrombin Receptor Antagonist for Clinical Event Reduction in Acute Coronary Syndrome (TRACER) trial (ClinicalTrials.gov number, NCT00527943). ${ }^{9}$ On the basis of data from that trial, the steering committee amended the main data-analysis plan to reorder the hierarchy of efficacy analyses, defining as the primary end point the composite of cardiovascular death, myocardial infarction, or stroke. The composite of cardiovascular death, myocardial infarction, stroke, or urgent coronary revascularization became the major secondary end point. Definitions of the components of these composite end points are provided in the Supplementary Appendix.

We assessed bleeding using the Global Use of Strategies to Open Occluded Coronary Arteries (GUSTO) classification system and the TIMI classification system, with GUSTO moderate or severe bleeding defined as the safety end point of primary interest. A clinical-events committee whose members were unaware of the study-group assignments adjudicated all components of the primary and major secondary efficacy end points and bleeding episodes.

\section{STATISTICAL ANALYSIS}

We calculated that 2279 events would be required to provide a power of at least $90 \%$ to detect a $15 \%$ relative risk reduction in the composite end point of cardiovascular death, myocardial infarction, stroke, or urgent coronary revascularization in the vorapaxar group, as compared with the placebo group. We estimated that 1400 events would be required to provide a power of at least $85 \%$ to detect the same relative treatment effect with respect to the composite end point of cardiovascular death, myocardial infarction, or stroke.
The primary efficacy analysis was conducted on an intention-to-treat basis among all patients who underwent randomization. After the data and safety monitoring board recommended discontinuation of vorapaxar in all patients with previous stroke, the protocol was amended to include supplementary evaluation of efficacy in patients who qualified for the trial with a diagnosis of myocardial infarction or peripheral arterial disease without a history of stroke before randomization. In addition, an analysis of efficacy that was restricted to patients with the qualifying diagnosis of myocardial infarction alone was specified in the original analysis plan before the board's recommendation.

The efficacy analyses were performed with the use of a Cox proportional-hazards model, with the study group and stratification factors at randomization as covariates. Cumulative event rates were calculated with the use of the Kaplan-Meier method at 3 years. Safety analyses were performed among patients who received one or more doses of a study drug and included events through 60 days after premature cessation of study therapy or 30 days after a final visit at the conclusion of the trial. On the basis of one planned interim analysis of efficacy, a P value of less than 0.049 was considered to indicate statistical significance for the final analysis, ${ }^{8}$ and a P value of less than 0.05 was deemed to indicate a significant interaction. All reported P values are two-sided.

\section{RESULTS}

\section{STUDY PATIENTS AND FOLLOW-UP}

From September 26, 2007, through November 13, 2009 , a total of 26,449 patients were enrolled in the trial. Of these, 13,225 were randomly assigned to receive vorapaxar, and 13,244 to receive placebo (Fig. S1 in the Supplementary Appendix).

Baseline characteristics of the patients are shown in Table 1. The qualifying diagnosis for enrollment was myocardial infarction in two thirds of the patients, stroke in 18\%, and peripheral arterial disease in $14 \%$. A total of $94 \%$ of the patients were treated with aspirin. At baseline, a thienopyridine was being administered in a majority of patients with a qualifying diagnosis of myocardial infarction but in only a minority of patients with a qualifying diagnosis of stroke or peripheral arterial disease. Only 177 patients $(0.7 \%)$ received prasugrel during the study.

The longest duration of follow-up was 49 


\begin{tabular}{|c|c|c|}
\hline Characteristic & $\begin{array}{l}\text { Vorapaxar } \\
(N=13,225)\end{array}$ & $\begin{array}{c}\text { Placebo } \\
(\mathrm{N}=13,224)\end{array}$ \\
\hline \multicolumn{3}{|l|}{ Demographic } \\
\hline \multicolumn{3}{|l|}{ Age } \\
\hline Median - yr & 61 & 61 \\
\hline Interquartile range $-\mathrm{yr}$ & $53-69$ & $53-69$ \\
\hline$\geq 75 \mathrm{yr}-$ no. (\%) & $1,514(11.4)$ & $1,506(11.4)$ \\
\hline Female sex — no. (\%) & $3,154(23.8)$ & $3,172(24.0)$ \\
\hline White race — no./total no. (\%) $†$ & $11,562 / 13,218(87.5)$ & $11,524 / 13,219(87.2)$ \\
\hline \multicolumn{3}{|l|}{ Qualifying type of atherosclerosis — no. (\%) } \\
\hline Myocardial infarction & $8,898(67.3)$ & $8,881(67.2)$ \\
\hline Ischemic stroke & $2,435(18.4)$ & $2,448(18.5)$ \\
\hline Peripheral arterial disease & $1,892(14.3)$ & $1,895(14.3)$ \\
\hline \multicolumn{3}{|l|}{ Selected clinical characteristics — no./total no. (\%) } \\
\hline Diabetes mellitus & $3,368 / 13,224(25.5)$ & $3,356 / 13,220(25.4)$ \\
\hline Hypertension & $9,047 / 13,225(68.4)$ & $9,127 / 13,219(69.0)$ \\
\hline Hyperlipidemia & $10,983 / 13,224(83.1)$ & $11,011 / 13,220(83.3)$ \\
\hline Current smoker & $2,748 / 13,225(20.8)$ & $2,750 / 13,220(20.8)$ \\
\hline Any coronary artery disease & $10,329 / 13,223(78.1)$ & $10,362 / 13,215(78.4)$ \\
\hline Previous coronary revascularization & $8,641 / 13,223(65.3)$ & $8,620 / 13,218(65.2)$ \\
\hline Any peripheral arterial disease & $2,901 / 13,224(21.9)$ & $2,944 / 13,218(22.3)$ \\
\hline Previous cerebrovascular event & $3,139 / 13,223(23.7)$ & $3,129 / 13,220(23.7)$ \\
\hline Estimated glomerular filtration rate $<60 \mathrm{ml} / \mathrm{min} / 1.73 \mathrm{~m}^{2} \div \mathrm{d}$ & $2,112 / 13,056(16.2)$ & $1,972 / 13,057(15.1)$ \\
\hline \multicolumn{3}{|l|}{ Use of antiplatelet agents — no./total no. (\%) } \\
\hline \multicolumn{3}{|l|}{ Qualifying myocardial infarction } \\
\hline Aspirin & $8,732 / 8,898(98.1)$ & $8,716 / 8,881(98.1)$ \\
\hline Thienopyridine & $6,934 / 8,898$ (77.9) & $6,960 / 8,881(78.4)$ \\
\hline \multicolumn{3}{|l|}{ Qualifying peripheral arterial disease } \\
\hline Aspirin & $1,661 / 1,892(87.8)$ & $1,671 / 1,895(88.2)$ \\
\hline Thienopyridine & $696 / 1,892(36.8)$ & $698 / 1,895(36.8)$ \\
\hline \multicolumn{3}{|l|}{ Qualifying stroke } \\
\hline Aspirin & $1,978 / 2,435(81.2)$ & $1,976 / 2,448(80.7)$ \\
\hline Thienopyridine & $574 / 2,435(23.6)$ & $580 / 2,448(23.7)$ \\
\hline Dipyridamole & $475 / 2,435(19.5)$ & $474 / 2,448(19.4)$ \\
\hline \multicolumn{3}{|l|}{ Use of other medications - no. (\%) } \\
\hline 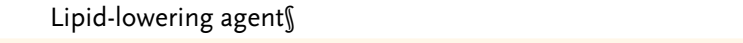 & $12,032(91.0)$ & $12,131(91.7)$ \\
\hline $\begin{array}{l}\text { Angiotensin-converting-enzyme inhibitor or angiotensin- } \\
\text { receptor blocker』 }\end{array}$ & $9,714(73.5)$ & $9,871(74.6)$ \\
\hline
\end{tabular}

* There were no significant differences between the two study groups, except as indicated.

$\dagger$ Race was self-reported.

The glomerular filtration rate was estimated with the use of the Modification of Diet in Renal Disease (MDRD) formula. $\int \mathrm{P}<0.05$.

months, with a median follow-up of 30 months (interquartile range, 24 to 36). Details of followup and loss to follow-up are provided in the Supplementary Appendix. The last date of patient contact was December 23, 2011, and the trial database was locked on January 9, 2012.

\section{EFFICACY END POINTS}

At 3 years, the primary end point of cardiovascular death, myocardial infarction, or stroke had occurred in 1028 patients $(9.3 \%)$ in the vorapaxar group, as compared with 1176 patients (10.5\%) in the placebo group (hazard ratio, 0.87 ; $95 \%$ confi- 
dence interval $[\mathrm{CI}], 0.80$ to $0.94 ; \mathrm{P}<0.001$ ) (Table 2 and Fig. 1A). The major secondary end point of cardiovascular death, myocardial infarction, stroke, or urgent coronary revascularization occurred in 1259 patients $(11.2 \%)$ in the vorapaxar group, as compared with 1417 patients (12.4\%) in the placebo group (hazard ratio, 0.88 ; $95 \% \mathrm{CI}, 0.82$ to 0.95 ; $\mathrm{P}=0.001$ ) (Table 2 and Fig. 1B). The rate of cardiovascular death or myocardial infarction was re- duced from $8.2 \%$ among patients in the placebo group to $7.3 \%$ among patients in the vorapaxar group ( $\mathrm{P}=0.002)$ (Table 2, and Fig. $\mathrm{S} 2$ in the Supplementary Appendix). Individual components of these composite end points are also shown in Table 2. The rate of death from any cause did not differ significantly between the vorapaxar group and the placebo group $(5.0 \%$ and $5.3 \%$, respectively; hazard ratio, 0.95 ; $95 \%$ CI, 0.85 to 1.07 ;

\begin{tabular}{|c|c|c|c|c|}
\hline \multirow[t]{2}{*}{ End Point } & Vorapaxar & Placebo & \multirow[t]{2}{*}{$\begin{array}{c}\text { Hazard Ratio } \\
(95 \% \mathrm{CI})\end{array}$} & \multirow[t]{2}{*}{ P Value } \\
\hline & \multicolumn{2}{|c|}{ number (percent) } & & \\
\hline Efficacy & 13,225 & 13,224 & & \\
\hline $\begin{array}{l}\text { Cardiovascular death, myocardial infarction, } \\
\text { or stroke }\end{array}$ & $1028(9.3)$ & $1176(10.5)$ & $0.87(0.80-0.94)$ & $<0.001$ \\
\hline $\begin{array}{l}\text { Cardiovascular death, myocardial infarction, stroke, } \\
\text { or urgent coronary revascularization }\end{array}$ & $1259(11.2)$ & $1417(12.4)$ & $0.88(0.82-0.95)$ & 0.001 \\
\hline Cardiovascular death or myocardial infarction & $789(7.3)$ & $913(8.2)$ & $0.86(0.78-0.94)$ & 0.002 \\
\hline Cardiovascular death & $285(2.7)$ & $319(3.0)$ & $0.89(0.76-1.04)$ & 0.15 \\
\hline Myocardial infarction & $564(5.2)$ & $673(6.1)$ & $0.83(0.74-0.93)$ & 0.001 \\
\hline \multicolumn{5}{|l|}{ Stroke } \\
\hline Any stroke & $315(2.8)$ & $324(2.8)$ & $0.97(0.83-1.14)$ & 0.73 \\
\hline Ischemic stroke & $250(2.2)$ & $294(2.6)$ & $0.85(0.72-1.01)$ & 0.06 \\
\hline Urgent coronary revascularization & $279(2.5)$ & $316(2.6)$ & $0.88(0.75-1.03)$ & 0.11 \\
\hline Death from any cause & $540(5.0)$ & $565(5.3)$ & $0.95(0.85-1.07)$ & 0.41 \\
\hline Bleeding & 13,186 & 13,166 & & \\
\hline GUSTO moderate or severe & $438(4.2)$ & $267(2.5)$ & $1.66(1.43-1.93)$ & $<0.001$ \\
\hline \multicolumn{5}{|l|}{ TIMI } \\
\hline Clinically significant & $1759(15.8)$ & $1241(11.1)$ & $1.46(1.36-1.57)$ & $<0.001$ \\
\hline Non-CABG-related major & $287(2.8)$ & $198(1.8)$ & $1.46(1.22-1.75)$ & $<0.001$ \\
\hline CABG-related major $†$ & $11(7.6)$ & $10(6.1)$ & $1.13(0.48-2.66)$ & 0.79 \\
\hline Fatal & $29(0.3)$ & $20(0.2)$ & $1.46(0.82-2.58)$ & 0.19 \\
\hline Intracranial & $102(1.0)$ & $53(0.5)$ & $1.94(1.39-2.70)$ & $<0.001$ \\
\hline Intracerebral & $89(0.8)$ & $41(0.4)$ & $2.19(1.51-3.17)$ & $<0.001$ \\
\hline Subdural or epidural & $12(0.1)$ & $10(0.1)$ & $1.20(0.52-2.79)$ & 0.67 \\
\hline Unknown & $1(<0.1)$ & $2(<0.1)$ & & \\
\hline Net clinical outcome & 13,186 & 13,166 & & \\
\hline $\begin{array}{c}\text { Cardiovascular death, myocardial infarction, stroke, } \\
\text { or GUSTO moderate or severe bleeding }\end{array}$ & $1315(11.7)$ & $1358(12.1)$ & $0.97(0.90-1.04)$ & 0.40 \\
\hline $\begin{array}{l}\text { Cardiovascular death, myocardial infarction, stroke, } \\
\text { urgent coronary revascularization, or } \\
\text { GUSTO moderate or severe bleeding }\end{array}$ & $1526(13.4)$ & $1593(14.0)$ & $0.96(0.89-1.02)$ & 0.20 \\
\hline $\begin{array}{l}\text { Death from any cause, myocardial infarction, stroke, } \\
\text { or GUSTO severe bleeding }\end{array}$ & $1322(11.9)$ & $1436(12.8)$ & $0.92(0.85-0.99)$ & 0.02 \\
\hline
\end{tabular}

* Percentages are cumulative Kaplan-Meier event rates at 3 years. The stroke component in all efficacy end points included all ischemic and hemorrhagic strokes, unless otherwise specified. Urgent coronary revascularization was defined by recurrent ischemia leading to urgent coronary revascularization. CABG denotes coronary-artery bypass grafting,

GUSTO Global Use of Strategies to Open Occluded Coronary Arteries, and TIMI Thrombolysis in Myocardial Infarction. $\uparrow$ CABG-related major bleeding was assessed in 175 patients in the vorapaxar group and 201 patients in the placebo group. 
$\mathrm{P}=0.41$ ). (Additional prespecified efficacy end points are shown in Tables S1 and S2 in the Supplementary Appendix.)

Among patients with no history of stroke, the primary end point occurred in $8.3 \%$ of patients in the vorapaxar group, as compared with $9.6 \%$ of those in the placebo group (hazard ratio, 0.84; $95 \%$ CI, 0.76 to 0.93 ; $\mathrm{P}<0.001$ ) (Table S3 in the Supplementary Appendix). There was no significant heterogeneity for the benefit of vorapaxar on

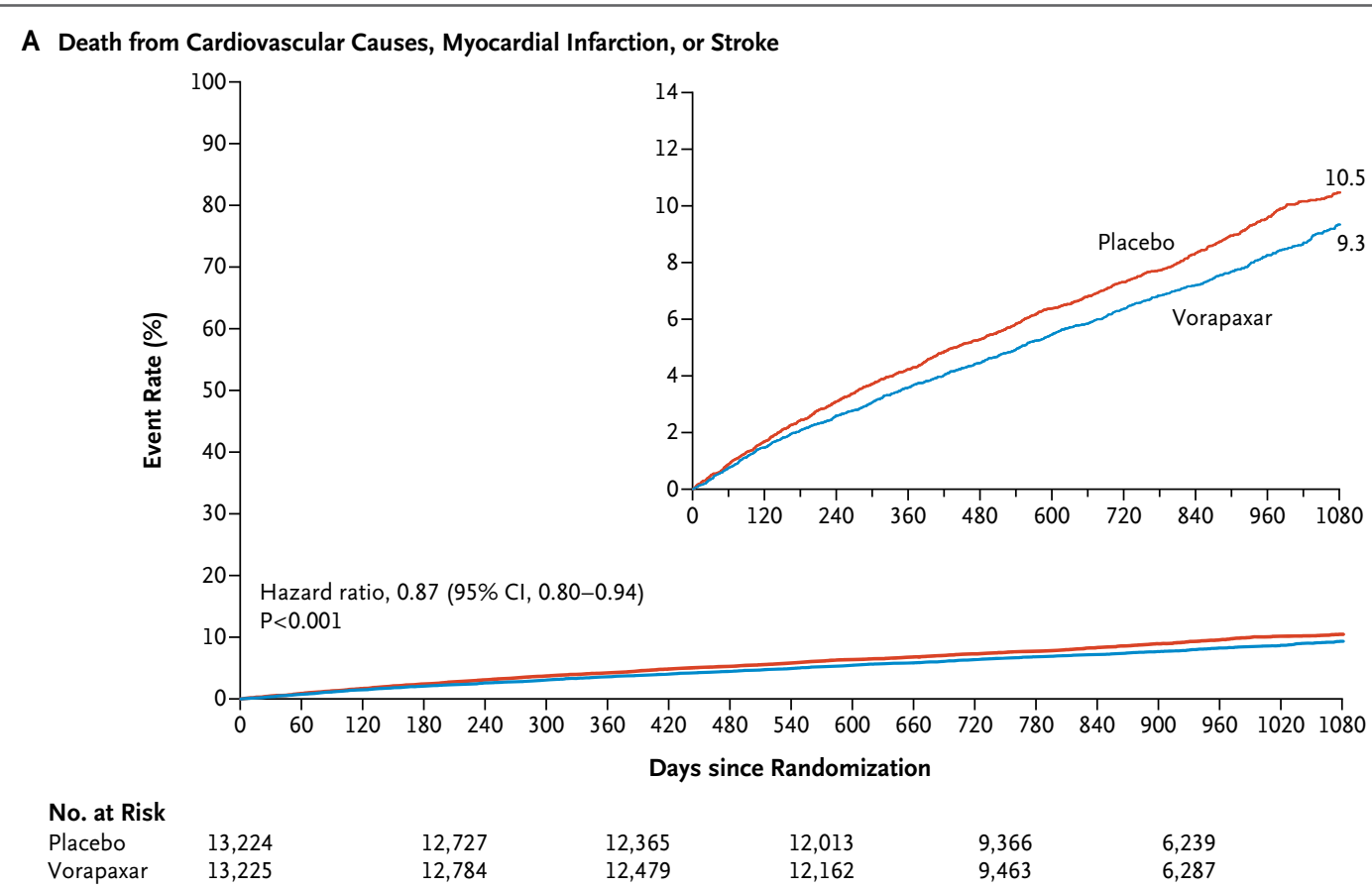

B Death from Cardiovascular Causes, Myocardial Infarction, Stroke, or Recurrent Ischemia Leading to Urgent Coronary Revascularization

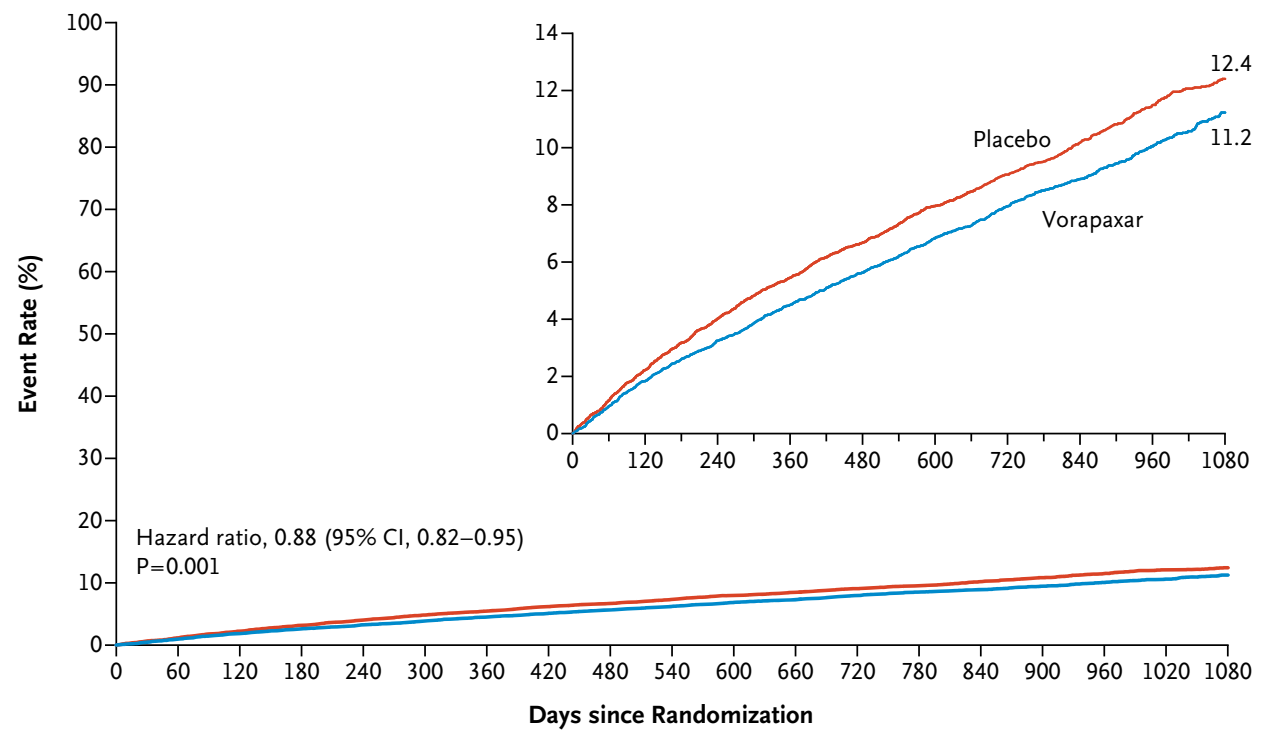

No. at Risk

Placebo

Figure 1. Kaplan-Meier Rates of Cardiovascular Events. 
the rate of cardiovascular death, myocardial infarction, or stroke across most of the major subgroups examined, including those defined according to the use or nonuse of a thienopyridine (Fig. S3 in the Supplementary Appendix). However, among patients who weighed less than $60 \mathrm{~kg}$, the use of vorapaxar did not have a favorable influence on this outcome ( $\mathrm{P}=0.03$ for interaction). Additional analyses of efficacy in subgroups that were defined according to the qualifying diagnosis of atherosclerosis (myocardial infarction, stroke, or peripheral arterial disease) and the presence or absence of a history of stroke are provided in Table S3 and Figure S3 in the Supplementary Appendix.

\section{SAFETY END POINTS}

The major safety end point of moderate or severe bleeding (according to GUSTO criteria) occurred in 438 patients ( $4.2 \%$ ) in the vorapaxar group, as compared with 267 patients $(2.5 \%)$ in the placebo group (hazard ratio, 1.66; 95\% CI, 1.43 to 1.93; $\mathrm{P}<0.001$ ) (Table 2 and Fig. 2A). There was no evidence of heterogeneity in the effect of vorapaxar on moderate or severe bleeding in major subgroups (Fig. S4 in the Supplementary Appendix). Rates of TIMI clinically significant bleeding (Fig. 2B) and rates of TIMI major bleeding not related to coronary-artery bypass grafting were also both significantly increased in the vorapaxar group as compared with the placebo group $(\mathrm{P}<0.001)$ (Table 2).

Overall, intracranial hemorrhage occurred in 102 patients $(1.0 \%)$ in the vorapaxar group, as compared with 53 patients $(0.5 \%)$ in the placebo group (hazard ratio, 1.94; 95\% CI, 1.39 to 2.70; $\mathrm{P}<0.001$ ) (Table 2, and Fig. S5 in the Supplementary Appendix). Fatal bleeding occurred in 29 patients $(0.3 \%)$ in the vorapaxar group, as compared with 20 patients $(0.2 \%)$ in the placebo group (hazard ratio, 1.46; $95 \% \mathrm{CI}, 0.82$ to $2.58 ; \mathrm{P}=0.19$ ). Among patients with a history of stroke, the rate of intracranial hemorrhage in the vorapaxar group was $2.4 \%$, as compared with $0.9 \%$ in the placebo group $(\mathrm{P}<0.001)$, with corresponding rates of fatal bleeding of $0.5 \%$ and $0.3 \%(\mathrm{P}=0.46)$ (Table $\mathrm{S} 3$ in the Supplementary Appendix). Among patients without a history of stroke, the rates of intracranial hemorrhage were lower in the two study groups ( $0.6 \%$ in the vorapaxar group and $0.4 \%$ in the placebo group, $\mathrm{P}=0.049$ ), as were the rates of fatal bleeding $(0.3 \%$ and $0.2 \%$, respectively; $\mathrm{P}=0.30)$.
Additional safety analyses are shown in Tables S3 and S4 in the Supplementary Appendix.

\section{NET CLINICAL OUTCOME}

A composite end point termed net clinical outcome, comprising the primary efficacy and safety end points, was prespecified (Table 2). The composite of cardiovascular death, myocardial infarction, stroke, or GUSTO moderate or severe bleeding occurred in 1315 patients $(11.7 \%)$ in the vorapaxar group and in 1358 patients (12.1\%) in the placebo group (hazard ratio, $0.97 ; 95 \% \mathrm{CI}, 0.90$ to 1.04; $\mathrm{P}=0.40$ ). Other net clinical outcomes in all patients and in patients without stroke are reported in Table 2, and Table S4 in the Supplementary Appendix.

DISCUSSION

In previous studies, it has not been established whether the addition of another antiplatelet agent to aspirin therapy would reduce the rate of thrombotic events in patients with stable atherosclerotic disease. ${ }^{4}$ In our large randomized trial, the PAR-1 antagonist vorapaxar significantly reduced the rate of cardiovascular death, myocardial infarction, or stroke in patients with a history of atherothrombosis who were receiving standard therapy. However, the reduction in cardiovascular events came at the cost of increased bleeding. This benefit and risk emerged early and continued to accrue throughout follow-up. Our findings show that inhibition of another platelet pathway in addition to that targeted by standard antiplatelet therapy for secondary prevention reduces the risk of recurrent thrombotic events in patients with previous atherothrombosis. This benefit was evident particularly in those whose qualifying diagnosis for participation in the trial was myocardial infarction.

The results of our study establish that interruption of the platelet-directed cellular actions of thrombin, suggested in preclinical studies to be pivotal to thrombosis,$^{10}$ translates into a clinical effect on major thrombotic events. In our study, antagonism of PAR-1 was complementary to inhibition of the thromboxane $\mathrm{A}_{2}$ and $\mathrm{P}_{2} \mathrm{Y}_{12}$-receptor pathways with aspirin and thienopyridines, with respect to the protective effect against recurrent thrombosis. We did not find evidence of significant heterogeneity of the effect of vorapaxar on the basis of treatment with clopidogrel. When vorapaxar was studied in the TRACER trial for the 


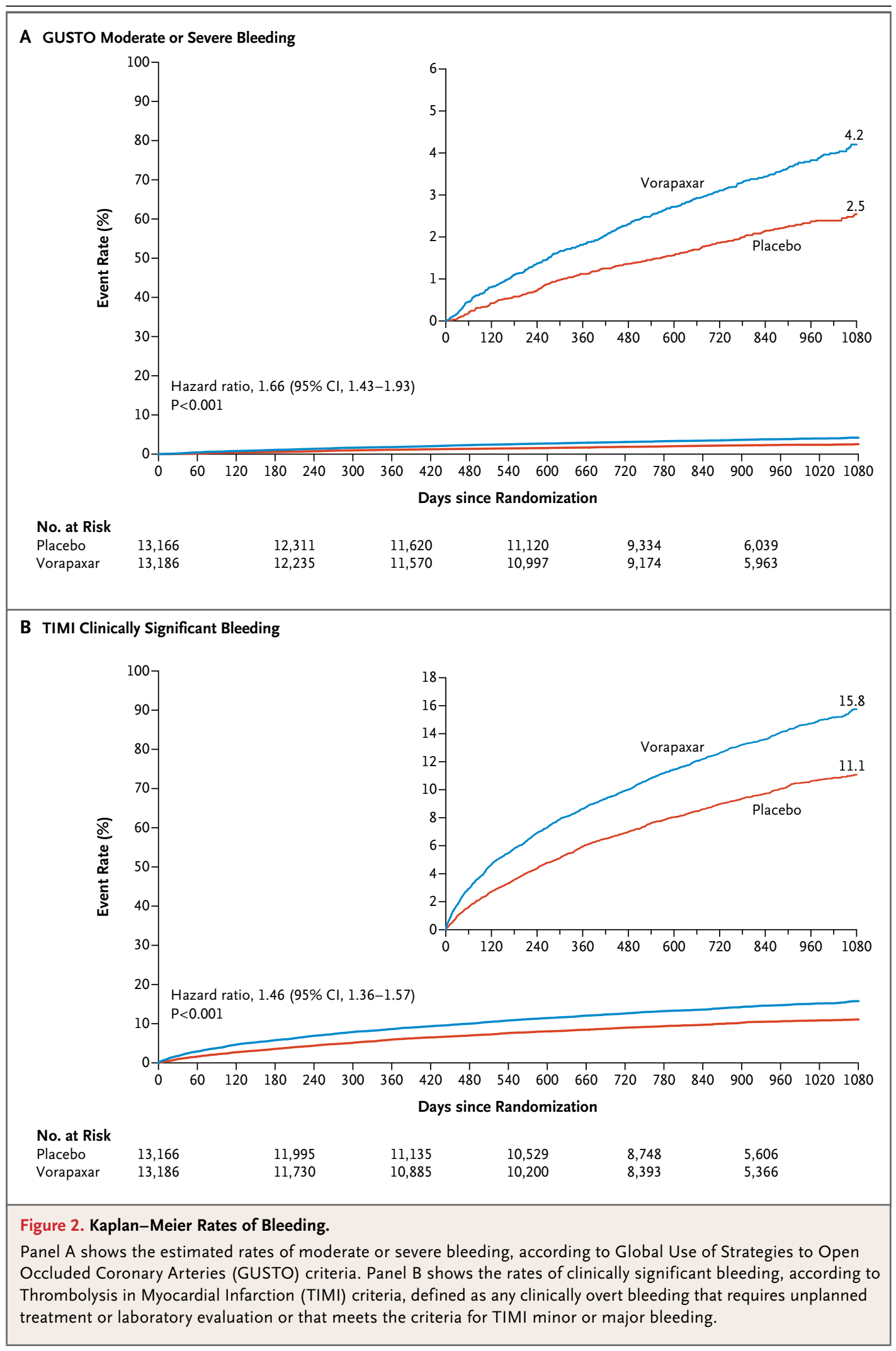

N ENGLJ MED 366;15 NEJM.ORG APRIL 12, 2012

The New England Journal of Medicine

Downloaded from nejm.org at AO San Paolo on March 5, 2020. For personal use only. No other uses without permission. 
management of acute coronary syndromes, there was a nonsignificant trend toward a reduction in the rate of cardiovascular death, myocardial infarction, stroke, recurrent ischemia with hospitalization, or urgent coronary revascularization and an exploratory finding of a reduction in the rate of cardiovascular death, myocardial infarction, or stroke, along with a significant increase in the risk of intracranial hemorrhage. ${ }^{9}$ Previous smaller phase 2 trials of vorapaxar ${ }^{11,12}$ and of atopaxar, ${ }^{13,14}$ another PAR-1 antagonist, have also revealed trends toward reductions in recurrent thrombotic events but without evidence of an increased risk of intracranial hemorrhage.

During the trial, the data and safety monitoring board recommended the discontinuation of vorapaxar in patients with a history of stroke on the basis of an excess of intracranial hemorrhage in such patients. We therefore carried out a separate analysis that was confined to patients without a history of stroke and found a significant benefit in this subgroup. In addition, in a prespecified analysis involving patients with a qualifying diagnosis of myocardial infarction, vorapaxar reduced the relative risk of the primary end point by $20 \%$. In the largest previous placebo-controlled trial of antiplatelet therapy for secondary prevention in patients with stable atherothrombosis or at high risk for vascular disease, clopidogrel plus aspirin was no better than aspirin alone in the overall cohort. ${ }^{4}$ Nevertheless, in an exploratory analysis involving 3846 patients with previous myocardial infarction in that trial, the addition of clopidogrel reduced the risk of cardiovascular death, myocardial infarction, or stroke by $23 \% .{ }^{15}$ Our trial involving a substantially larger number of patients with a history of myocardial infarction shows that the addition of another antiplatelet agent to aspirin therapy over the long term can achieve further reductions in recurrent atherothrombotic events in this population.

The reduction in thrombotic events with vorapaxar came with significant increases in bleeding. Although our findings show that additional reductions in atherothrombosis can be achieved, this benefit must be weighed against the increase in bleeding risk. In the overall trial cohort, there was no significant between-group difference in the prespecified net clinical outcome (including both thrombotic end points and moderate or severe bleeding). However, weighed against the risk of severe bleeding, particularly in patients without a history of stroke, the net clinical outcome was improved in patients receiving vorapaxar.

The results of our study also add to the accumulating evidence of a heightened risk of intracranial hemorrhage among patients with a history of stroke who are treated with potent antiplatelet therapy. ${ }^{2,16,17}$ As with the use of third-generation

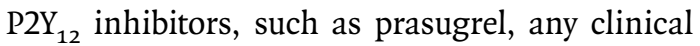
use of vorapaxar would have to be based on an appropriate selection of patients, with the risk of bleeding balanced against that of recurrent thrombotic events. We have previously identified criteria for selecting patients who are more likely to have improved net clinical outcomes with potent antiplatelet therapy. ${ }^{2,18}$ In our study, the relative risk of bleeding, including intracranial hemorrhage, showed a similar significant increase in the vorapaxar group among patients with and those without a history of stroke. However, the absolute rate of intracranial hemorrhage $(0.2 \%$ per year) among patients without a history of stroke was substantially lower than that among patients with such a history $(0.8 \%$ per year). The rate of fatal bleeding was not significantly increased in the vorapaxar group.

In conclusion, the addition of vorapaxar to standard therapy reduced the risk of cardiovascular death, myocardial infarction, or stroke among patients with stable atherosclerosis, a benefit that was most apparent in patients with a history of myocardial infarction. Vorapaxar also increased the risk of moderate or severe bleeding, including intracranial hemorrhage, with the latter occurring most frequently in patients with a history of stroke.

Supported by Merck.

Disclosure forms provided by the authors are available with the full text of this article at NEJM.org.

The authors' affiliations are as follows: the Thrombolysis in Myocardial Infarction (TIMI) Study Group, Cardiovascular Division, Department of Medicine, Brigham and Women's Hospital, Boston (D.A.M., E.B., M.P.B., M.P.F., B.M.S., S.D.W., S.A.M.); the Institute for Neurological Research, FLENI, Buenos Aires (S.F.A.); Milpark Hospital, Johannesburg (A.J.D.); the Division of Cardiovascular Research, University of Edinburgh, Edinburgh (K.A.A.F.); Merck Research Laboratories, Rahway, NJ (L.J.L., X.L., J. Strony); the Heart Institute (InCor)-University of São Paulo Medical School, São Paulo (J.C.N.); Canisius-Wilhelmina Hospital, Nijmegen, the Netherlands (A.J.O.O.); Instituto de Investigaciones Clínicas Rosario, Rosario, Argentina (E.P.); University Hospital Brno, Masaryk University, Brno, Czech Republic (J. Spinar); and Montreal Heart Institute and University of Montreal, Montreal (P.T.). 
REFERENCES

1. Yusuf S, Zhao F, Mehta SR, Chrolavicius S, Tognoni G, Fox KK. Effects of clopidogrel in addition to aspirin in patients with acute coronary syndromes without ST-segment elevation. N Engl J Med 2001;345:494-502. [Errata, N Engl J Med 2001;345:1506, 1716.]

2. Wiviott SD, Braunwald E, McCabe $\mathrm{CH}$, et al. Prasugrel versus clopidogrel in patients with acute coronary syndromes. N Engl J Med 2007;357:2001-15.

3. Wallentin L, Becker RC, Budaj A, et al. Ticagrelor versus clopidogrel in patients with acute coronary syndromes. N Engl J Med 2009;361:1045-57.

4. Bhatt DL, Fox KA, Hacke W, et al. Clopidogrel and aspirin versus aspirin alone for the prevention of atherothrombotic events. N Engl J Med 2006;354:1706-17.

5. Leger AJ, Covic L, Kuliopulos A. Protease-activated receptors in cardiovascular diseases. Circulation 2006;114:1070-7. 6. Chackalamannil S, Xia Y, Greenlee WJ, et al. Discovery of potent orally active thrombin receptor (protease activated re ceptor 1) antagonists as novel antithrombotic agents. J Med Chem 2005;48:5884-7 7. Chackalamannil S. Thrombin receptor (protease activated receptor-1) antagonists as potent antithrombotic agents with strong antiplatelet effects. J Med Chem 2006;49:5389-403.

8. Morrow DA, Scirica BM, Fox KA, et al.
Evaluation of a novel antiplatelet agent for secondary prevention in patients with a history of atherosclerotic disease: design and rationale for the Thrombin-Receptor Antagonist in Secondary Prevention of Atherothrombotic Ischemic Events (TRA 2P)-TIMI 50 trial. Am Heart J 2009;158(3): 335.e3-341.e3.

9. Tricoci $\mathrm{P}$, Huang $\mathrm{Z}$, Held $\mathrm{C}$, et al. Thrombin-receptor antagonist vorapaxar in acute coronary syndromes. N Engl J Med 2012;366:20-33.

10. Coughlin SR. Thrombin signalling and protease-activated receptors. Nature 2000;407:258-64.

11. Becker RC, Moliterno DJ, Jennings LK, et al. Safety and tolerability of SCH 530348 in patients undergoing non-urgent percutaneous coronary intervention: a randomised, double-blind, placebo-controlled phase II study. Lancet 2009;373:919-28.

12. Goto S, Yamaguchi T, Ikeda Y, Kato K, Yamaguchi H, Jensen P. Safety and exploratory efficacy of the novel thrombin receptor (PAR-1) antagonist SCH530348 for non-ST-segment elevation acute coronary syndrome. J Atheroscler Thromb 2010;17: 156-64.

13. O'Donoghue ML, Bhatt DL, Wiviott SD, et al. Safety and tolerability of atopaxar in the treatment of patients with acute coronary syndromes: the Lessons From Antagonizing the Cellular Effects of
Thrombin-Acute Coronary Syndromes trial. Circulation 2011;123:1843-53.

14. Wiviott SD, Flather MD, O'Donoghue $\mathrm{ML}$, et al. Randomized trial of atopaxar in the treatment of patients with coronary artery disease: the Lessons From Antagonizing the Cellular Effect of ThrombinCoronary Artery Disease trial. Circulation 2011;123:1854-63.

15. Bhatt DL, Flather MD, Hacke W, et al. Patients with prior myocardial infarction, stroke, or symptomatic peripheral arterial disease in the CHARISMA trial. J Am Coll Cardiol 2007;49:1982-8.

16. Sacco RL, Diener H-C, Yusuf S, et al. Aspirin and extended-release dipyridamole versus clopidogrel for recurrent stroke. N Engl J Med 2008;359:1238-51.

17. Diener H-C, Bogousslavsky J, Brass LM, et al. Aspirin and clopidogrel compared with clopidogrel alone after recent ischaemic stroke or transient ischaemic attack in high-risk patients (MATCH): randomised, double-blind, placebo-controlled trial. Lancet 2004;364:331-7.

18. Wiviott SD, Desai N, Murphy SA, et al. Efficacy and safety of intensive antiplatelet therapy with prasugrel from TRITONTIMI 38 in a core clinical cohort defined by worldwide regulatory agencies. Am J Cardiol 2011;108:905-11.

Copyright (C) 2012 Massachusetts Medical Society. 